\title{
GLOBAL CONTROL OF THE BARCELONA SEWERAGE SYSTEM FOR ENVIRONMENT PROTECTION
}

\author{
G. Cembrano $^{\circ}$, J. Figueraso*, J. Quevedo*, V. Puig*, M. Salamero ${ }^{\#}$, J. Martï \\ Instituto de Robótica e Informática Industrial (UPC-CSIC) \\ Parc Tecnolòic de Barcelona, Edifici $U$ \\ Llorens i Artigas 4-6, Planta 2 - 08028 Barcelona \\ gcembrano@iri.upc.es \\ *Dept. Enginyeria de Sistemes, Automàtica i Informàtica Industrial \\ Universidad Politécnica de Cataluña - Campus de Terrassa \\ Rambla Sant Nebridi, 10 - 08222 Terrassa \\ ${ }^{\#}$ Clavegueram de Barcelona S.A. \\ Acer, 16 - 08038 Barcelona
}

\begin{abstract}
This paper deals with the use of optimal control techniques in the Barcelona urban drainage system, which includes flow-diversion gates, detention tanks and a telemetry/telecontrol system. Optimal control is used to provide control strategies reducing the events of flooding and polluting discharges to the environment. The influence of rain prediction on the optimal control results is analysed in this paper. Copyright (C) 2002 IFAC
\end{abstract}

Keywords: Real-time control, Urban drainage, Predictive control, Optimal control, Environment protection.

\section{INTRODUCTION}

The increasing concern of society with sustainable development raises, among other crucial subjects, the issue of water management in urban areas, including not only water supply but also the disposal of effluents to the receiving environment (Krebs and Larsen, 1997; Price, 2000).

Urban drainage systems are in charge of carrying urban wastewater and rainwater to one or more terminal points, where it is treated and/or released to the receiving environment. Combined sewer systems carry rain- and wastewater together. In many cities where the conurbation has been growing fast and stormy rains are frequent, the existing combined sewer systems are unable to carry all the rain- and wastewater to the treatment plants when highintensity rain occurs. This results in flooding of certain areas and combined sewer overflows (CSO) which release untreated rain- and waste-water to the receiving waters.

Advanced urban drainage is an attempt to solve these problems. It involves, on the one hand, the improvement of infrastructure -the construction of detention tanks, flow-diversion gates, pumps, sluice gates, etc.- and, on the other, the installation of a telemetry and supervisory control system which can execute real-time control. The telemetry system contains rain-gauges distributed in several areas of the city, as well as flow or level meter (limnimeters) and quality meters in the main sewers, which periodically send information to a central dispatch. Real-time control implies that the operation flow regulation elements, such as gates and pumps, is based on the real-time process data supplied by telemetry. 
Real-time control of an urban drainage system may be local or global. When local control is applied, flow regulation devices use only measurements taken at its specific location. Whereas this control structure is applicable in many simple cases, in a large city, with a strongly interconnected sewerage network and a complex network of actuators and sensors, it may not be the most efficient alternative. Conversely, global control, which computes control actions taking into account real-time measurements all through the network, is likely to make the best use of the infrastructure capacity and all the available sensor information.

Real-time control in urban drainage systems has been the object of research for the last 30 years, but only very few cities have actually implemented such systems in North America and Europe. Most of these have implemented local reactive control rather than global control. (More detailed information on the specific applications is available from the Group on Real Time Control of Urban Storm Drainage at their web site: http://web.tiscali.it/RTCUSD).

The Barcelona urban drainage network contains approximately 1600 kilometers of sewers carrying a daily volume of some $300 \mathrm{~m}^{3}$ wastewater in dry weather and the rainwater an average precipitation of $600 \mathrm{~mm}$ a year. It includes 3 detention tanks (other 3 are under construction, to be inaugurated by 2002) with a total of $273000 \mathrm{~m}^{3}$ capacity and 16 gates are currently in operation. A telemetry network containing 24 rain gauges, more than 100 limnimeters and a few quality sensors connected to a Supervisory Control and Data Acquisition system (SCADA) have been in operation since 1994. Local reactive control of the flow regulation devices in the network has been in use since 1998 .

With the objective of reducing flooding and CSO and in order to take full advantage of the storage capacity of the urban drainage network and the treatment plants, a collaborative project for the implementation of real-time global control in the whole Barcelona network is underway, scheduled to be in operation by March 2003. An off-line prototype of the system has been recently developed and tested, as reported in (Cembrano et al., 2001). The predictive control setup used in this prototype has been tested further, in order to assess the validity of the obtained optimal strategies and the influence of the prediction approach on the fulfilment of the control goals. These new results are the object of this communication.

The fundamentals of global optimal control in urban drainage systems are outlined in Section 2; Section 3 deals with predictive control approach chosen in this application and Section 4 describes the experiments with different prediction schemes. Conclusions and future developments are summarized in section 5 .

\section{OPERATIONAL MODEL OF AN URBAN DRAINAGE SYSTEM}

\subsection{Fundamentals}

An operational model of an urban drainage system is a set of equations which provide a fast approximate evaluation of the hydraulic variables of the network and its response to control actions at the gates. This type of model is useful for the computation of optimal strategies, because it makes it possible to evaluate a large number of control actions in a short computation time.

This type of model is usually referred to as a "conceptual" or "transfer function" model (Norreys and Cluckie, 1997; Cluckie, Lane and and Yuan, 1999; Nguyen, Loong and Woo,2000), or virtualreservoir model (Ballester, 1998).

The sewage network is modeled through a simplified graph relating the main sewers and set of virtual and real reservoirs. A virtual reservoir is an aggregation of a catchment of the sewage network which approximates the hydraulics of rain, runoff and sewage water retention thereof. The hydraulics of virtual reservoirs are linearized so that, in discrete time:

$$
x^{k+1}=f\left(x^{k}, u^{k}, w^{k}\right)
$$

where:

$u$ represents a vector of control variables related to gate positioning (e.g. flow through the gate), $x$ is a vector of observable states: stored volumes in reservoirs (real and virtual) and flows (or water levels) in main sewers, $w$ is a disturbance vector containing rainfall intensities in the different catchments, $f$ is a linear function expressing the mass balance of rain intake, sewer flow and reservoir volumes in the networks. Its structure depends on the topology of the network and its parameters must be estimated using real data from the sensors in the network. In the application described in this paper, adaptive, on-line parameter identification is used and superindexes $k$ indicate time intervals.

\subsection{Modelling the Barcelona Test Catchment}

An important area of Barcelona (Fig. 1) has been selected as a test site to analyse the results of this work. It is comprised of 11 virtual reservoirs, one real detention tank with its inlet and outlet gates, 18 main sewers, 3 flow-diversion gates and 5 overflow diversion devices (Fig. 2). It includes the connections to the sea and to the wastewater treatment plant, as well as superficial flows during flooding events. 


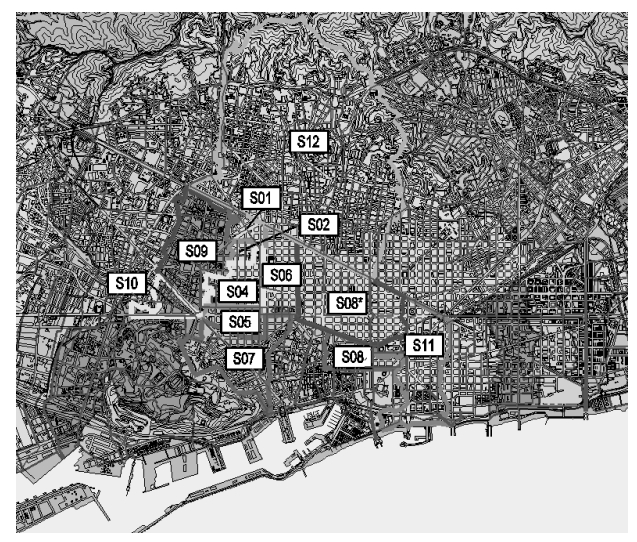

Fig 1. Selected Area of Barcelona

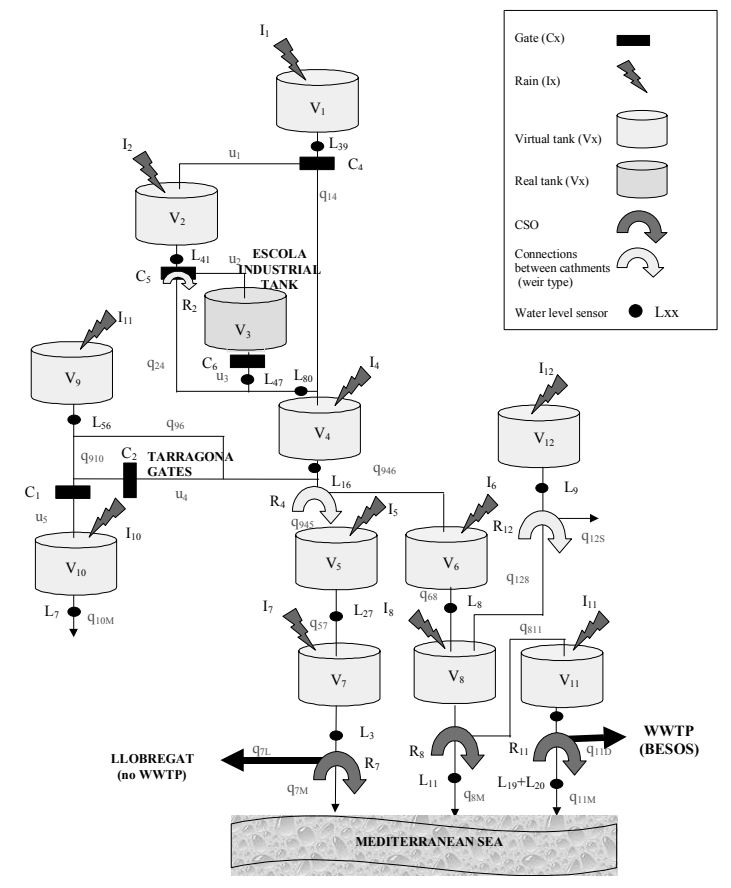

Fig. 2. The Reservoir Model of the Selected Area of Barcelona

After the selection area of the benchmark, all the parameters of this model have been estimated with historic data of water level sensors and rain gauges, using the MATLAB System Identification Toolbox. Fig. 3 shows the comparison between the real data of limnimeter $\mathrm{L}_{39}$ and the prediction of the first virtual reservoir model for a 30-minute prediction horizon, which is also the optimal predictive control horizon. The prediction of the model shows a good adjustment for short, but logically, the discrepancies of the model output increase when the prediction horizon increases. For this study the rainfall intensities have been kept constant during the prediction horizon.

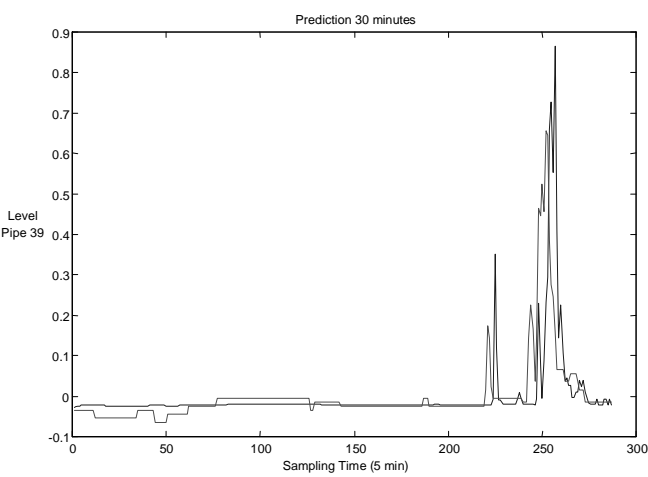

Fig. 3. 30-minute prediction (blue) versus real measures (red) for limnimeter $\mathrm{L}_{39}$

\section{OPTIMAL CONTROL IN URBAN DRAINAGE}

\subsection{Optimal Control Problem}

The optimal control goals in urban drainage systems are generally concerned with environmental protection, in particular, flood prevention and minimization of CSO. The objective of applying optimal control is to compute, ahead of time, feasible strategies for the actuators in the network which produce the best admissible states of the network, in terms of these objectives, during a certain horizon. The control period must be defined taking into account the telemetry system sampling time and the time constants of the actuators in the network. The optimization horizon must be adequately considering the hydraulic time constants of storm water evacuation, reservoir depletion and/or other factors affecting the urban drainage management.

The computed strategies are based on rain forecasts through the optimization horizon, so that the predictability horizon of the rain data, a measure of how long ahead in time forecasts have acceptable degrees of confidence, bounds the choice of the optimization horizon.

Hence, the control problem is stated as an optimization of a performance index $\boldsymbol{J}$, a summation over the optimization horizon $\boldsymbol{N}$ (expressed in number of control intervals) of a function of states and controls $\boldsymbol{h}$, which evaluates the fulfilment of the control goals and with a linear on non-linear expression.

\subsection{Optimal Control Prototype for Barcelona}

In the Barcelona prototype, linear and non-linear penalty functions have been used to impose high costs to strategies which produce flooding or CSO.

$$
J=\sum_{N} h(x, u)
$$

The optimization must be performed with respect to the control variables $\boldsymbol{u}$ and it is subject to a set of constraints, namely: 
- linear equality equations expressing the mass balance, including rain intake, in the reservoirs and the sewers,

- non-linear equality flow equations related to the overflow devices,

- and bound constraints on the operative range of gates, on the allowable flows through the sewers and on maximum reservoir capacities

which are written as follows:

$$
\begin{aligned}
& A x+B u+C w=0 \\
& g(x)=0 \\
& x_{\text {min }} \leq x \leq x_{\text {mac }} \\
& u_{\text {min }} \leq u \leq u_{\max }
\end{aligned}
$$

where $A, B$ and $C$ are the coefficient matrices affecting $x, u$, and $w$ in the mass balance equations, $g$ is a nonlinear function of the flow and $x_{\min }, x_{\max }$, $u_{\min }$ and $u_{\max }$ are bounds on the states and the controls respectively.

In the Barcelona prototype, a sampling time (and control interval) of 5 minutes is used, based on actuator response times and on the scanning time of the telemetry system. An optimization horizon of 30 minutes is chosen, taking into account the time constants of reservoir depletion and the average transportation time from the inlets to the terminal points of the drainage system

\subsection{Results of the Optimal Control Prototype}

The optimal control strategies obtained with the above-described system were shown to produce significant improvements on flooding and CSO minimization, as well as in the use of the treatment plant capacity (Cembrano et al. 2002).

As an illustration Table 1 shows the comparison of the total flooding, CSO and treatment plant volumes in three situations: without a detention tank, with the detention tank but using it as a passive element (inlet and outlet gates open at all times) and with optimal control.

\begin{tabular}{|l|r|r|r|}
\multicolumn{4}{c|}{ Heavy-Rain Episode (14/09/99) } \\
\cline { 2 - 4 } & $\begin{array}{c}\text { Passive no } \\
\text { tank }\end{array}$ & $\begin{array}{c}\text { Passive with } \\
\text { tank }\end{array}$ & Optimized \\
\hline Flooding, m $^{3}$ & 178647 & 138582 & 130293 \\
\hline Improvement, \% & & $\mathbf{2 2 , 4 3}$ & $\mathbf{2 7 , 0 7}$ \\
\hline Treated Water, $\mathbf{m}^{3}$ & 117375 & 132900 & 134307 \\
\hline Improvement, \% & & $\mathbf{1 3 , 2 3}$ & $\mathbf{1 4 , 4 3}$ \\
\hline Flood & 878817 & 867378 & 865509 \\
\hline Improvement, \% & & $\mathbf{1 , 3 0}$ & $\mathbf{1 , 5 1}$ \\
\hline
\end{tabular}

Table 1. Results with heavy-rain scenario

From Table 1, it can be observed that the use of a detention reservoir produces improvements in flooding (most importantly) and CSO. The use of optimal control produces significant additional improvements in both concepts (a total of over $30 \%$ flood reduction in a heavy rain scenario and $3 \%$ CSO reduction during a medium-intensity rain). Additionally, the use of the wastewater treatment plant is improved .

When analyzing these results, it is important to remark that this prototype network, with just one $27.000 \mathrm{~m}^{3}$ detention tank has a limited capacity to store storm water in heavy-rain conditions. Moreover, the reservoir and the flow-diversion gates are very distant from the CSO sites, so that their ability to control CSO is also limited. Even with these severe limitations, the results show the potential of using optimal control in the Barcelona sewer network. The extension of this concept to the complete network (with some $270.000 \mathrm{~m}^{3}$ storage capacity and a larger number of controls is currently ongoing.

\section{EXPERIMENTS WITH DIFFERENT PREDICTION MODES}

\subsection{Object of the experiments}

The first results of the Barcelona prototype were obtained using a constant-rain prediction. The rain intensity is considered constant over the 30-minute optimization horizon, equal to the current reading of the rain gauges. However, in the predictive control scheme used in this work, the optimal strategy is recomputed every five minutes with new sensor readings, so that only the first five-minute set-point of each 30-minute strategy is actually used.

Then the issue of the dependence of the results on the validity of the rain predictions was considered. Could the global results in terms of urban drainage management parameters be improved by providing a better rain prediction?. The experiments used 3 different prediction modes. In all cases, the sensor data and the optimal strategies are updated every five minutes. The main experiments are as follows:

- Experiment 1: "Perfect prediction". Real rain intensity data are fed to the optimal control program, with the intention of obtaining the "ideal" performance, against which the 3 prediction modes will be tested.

- Experiment 2: "Constant prediction". The rain intensity is considered constant over the halfhour optimization horizon.

- Experiment 3: “Worst-case prediction". The current and the previous rain intensity readings are considered and a numeric derivative is computed. When the derivative is positive, the next 30-minute predictions are computed keeping this derivative constant. If this derivative is zero or negative, then, the rain prediction is kept constant 
- Experiment 4: "Best-case prediction": The current reading is considered valid for five minutes and the rain intensity prediction is zero for the rest of the prediction horizon

Two different scenarios were chosen: a heavy rain with average return period of 4 years (and peak return periods of 12 years, used also in Table 1) and a moderate rain $(0.7$-year and 12-year average and peak return period respectively). In all cases, the comparison is established using the global urban drainage management evaluators, namely, the total flood volume, FV, the total CSO volume and the total volume sent to the treatment plant, TPV, over the complete rain scenario (lasting approximately 4 hours in each case).

\subsection{Results}

The results of these experiments in the heavy-rain scenario are summarized in Table 2 and those obtained for the moderate-rain scenario are contained in Table 3.

\begin{tabular}{|l|l|l|l|l|}
\hline & Exp.1 & Exp 2 & Exp.3 & Exp.4 \\
\hline FV & 133113 & 130293 & 134139 & 128385 \\
\hline CSO & 865458 & 865509 & 865374 & 865929 \\
\hline TPV & 133782 & 134307 & 133782 & 134295 \\
\hline
\end{tabular}

Table 2. Global results in a heavy-rain scenario (volumes in $\mathrm{m}^{3}$ )

\begin{tabular}{|l|l|l|l|l|}
\hline & Exp.1 & Exp.2 & Exp.3 & Exp.4 \\
\hline CSO & 417849 & 418455 & 419625 & 419577 \\
\hline TPV & 155937 & 155862 & 155025 & 157605 \\
\hline
\end{tabular}

$\underline{\text { Table 3. Global results in a moderate-rain scenario }}$ (volumes in $\mathrm{m}^{3}$ )

These global results in both rain conditions show that the constant-rain strategy gives only slightly poorer results than their ideal values, i.e., those obtained with the "perfect prediction" mode. This is so because of the adopted predictive-control scheme, in which the rain data and the strategies are updated every five minutes with the new real values.

Additionally, the "best-case" prediction mode gives better results in terms of smaller flood volume for the heavy rain situation In the moderate-rain scenario, the constant-rain-prediction strategy gives a CSO values that is closer to the ideal one (the perfect-prediction strategy), but the "best-case" prediction strategy performs better in terms of a larger volume sent to the treatment plant then any of the other strategies. The "worst-case" prediction has the poorest performance for both rain situations.

An additional factor for evaluating the different prediction strategies is the usage of the detention tank's storage capacity produced by the control strategies obtained with these prediction modes. Figures $4 \mathrm{a}, 4 \mathrm{~b}, 5 \mathrm{a}$ and $5 \mathrm{~b}$ show the evolution of the detention tank volume with the strategies obtained using all four prediction modes, in the same two rain scenarios. In both cases, the strategy that best takes advantage of the storage capacity (larger value of the area under the curve) is, not surprisingly, the one using real data. However, the best-case-prediction strategy attains an almost equally good use of the tank for the heavy-rain case. In the moderate-rain scenario both the constant-rain and the "best-case" strategies give a tank usage that is comparable to that of the "perfect-prediction" strategy. The "worstcase" prediction strategy gives a poorer usage of the tank in both situations.

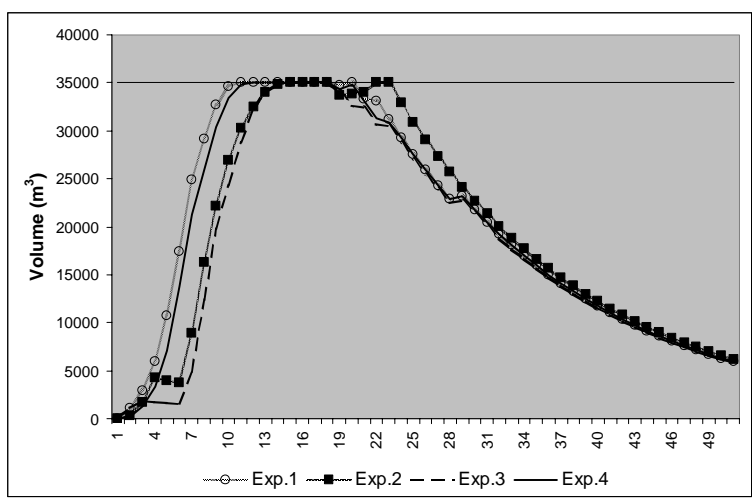

Fig.4a Detention tank usage in heavy-rain scenario

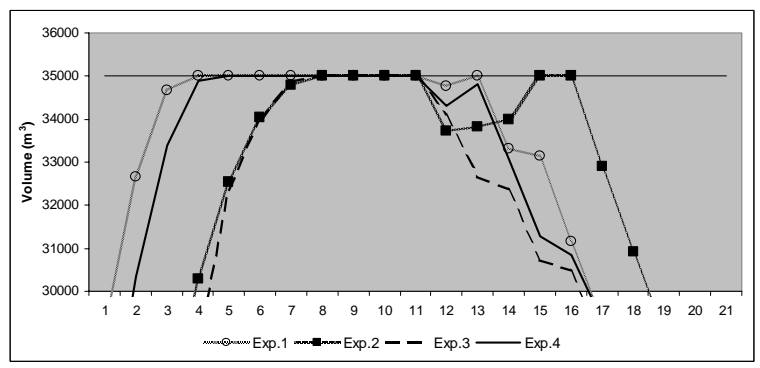

Fig.4b Detention tank usage in heavy-rain scenario (detail)

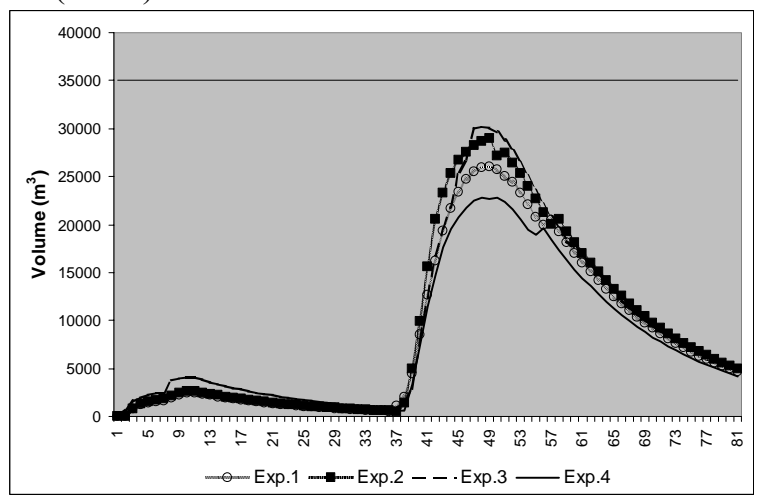

Fig.5a Detention tank usage in moderate-rain scenario 


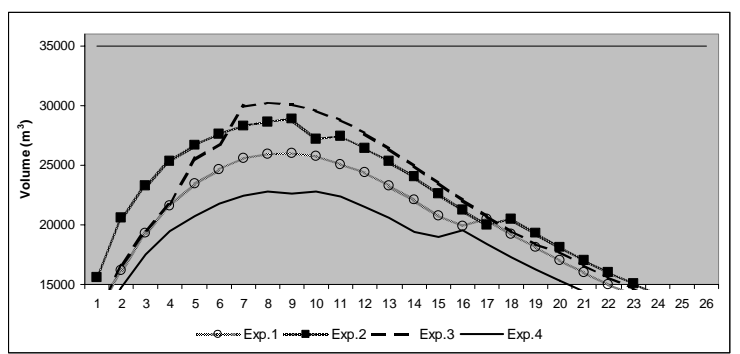

Fig.5b Detention tank usage in moderate-rain scenario (detail)

From these experiments, it may be concluded that the "best-case" and the "constant-rain" prediction modes perform well under the adopted predictive control set-up.

\section{CONCLUSIONS}

An off-line prototype of global optimal control of the Barcelona sewerage network, which was earlier shown to provide significant improvement in environmental protection factors, has been tested with different rain-prediction modes. The object of the test was to evaluate the influence of the prediction mode on the performance of the optimal control strategies. The experiments compare the results obtained using three different prediction modes to those obtained when real data of the rain scenario are used to generate the control strategies.

The "constant-rain" and the "best-case" prediction modes give performances close to the ideal values obtained using real data, whereas the more conservative "worst-case" prediction mode performs poorly.

The on-line implementation of the real-time optimal control in the area covered by the prototype is currently underway in the Barcelona urban drainage system. After this system has been validated in real operation, the system will be extended to the complete network.

\section{FURTHER RESEARCH}

Further improvements in rain-prediction could be introduced in order to obtain better results in the optimal control than those presented in Section 4 of this paper obtained using the proposed simple rainprediction strategies. In Toth et al. (2000), quantitative forecasting methods based on ARMA models, Neural Networks and K-Nearest-Neighbour are used and compared in the context of real-time flood forecasting combining them with a lumped conceptual rainfall-runoff transformation model. This study indicates that the considered time-series analysis techniques provide an improvement in the flood forecasting accuracy with respect to the use of intuitive heuristic rainfall prediction approaches, as the ones proposed in this paper, even if the rainfall forecasting measures indicate only a weak to moderate relationship between forecasted and observed values. Rain prediction techniques proposed in this reference will be tested in the context of the optimal control of Barcelona urban drainage system against those presented in this paper.

\section{ACKNOWLEDGEMENTS}

This work has been partly funded by the CICYT (Project COO1999 AX072) Authors J. Quevedo and V. Puig are members of LEA-SICA (Associated European Laboratory on Intelligent Systems and Advanced Control)

The contribution of the CLABSA staff in supplying data and validating the experiments is gratefully acknowledged.

\section{REFERENCES}

Ballester, J.L., Martí, J. and M. Salamero (1998). "Control de Compuertas de Derivación en la Red de Alcantarillado de Barcelona”. Ingeniería del Agua. Vol. 5, No 4, pp. 37-46.

Camacho, E.F. and C. Bordons (1999). "Model Predictive Control" in Advanced Textbooks in Control and Signal Processing. Springer. London.

Cembrano, G., Quevedo, Salamero, M., Puig, V., Figueras, J. and J. Martí (2002). "Optimal Control of Urban Drainage Systems". Control Engineering Practice (in press). Elsevier. Great Britan.

Cluckie, I D., Lane, A. and J. Yuan (1999). "Modelling Large Urban Drainage Systems" in Real Time Water Science and Technology Vol. 39, No.4 pp 21-28. Elsevier. Great Britain.

Krebs, P. and A. Larsen (1997). "Guiding the Development of Urban Drainage Systems by Sustainability Criteria". Water Science and Technology Vol. 35, No.9 pp 89-98. Elsevier. Great Britain

Norreys, R and I. Cluckie (1997). "A Novel Approach to Real-Time Modelling of Large Urban Drainage Systems". Water Science and Technology Vol. 36, No.8-9 pp 19-24. Elsevier. Great Britain

Price, R. K (2000). "Hydroinformatics and Urban Drainage: An Agenda for the $21^{\text {st }}$ Century" Journal of Hydroinformatics Vol.2 No.2 IWA Publishing.

Toth, E., Brath, A. and A. Montanari (2000). "Comparison of short-term rainfall prediction models for real-time flood forecasting" Journal of Hydrology, 239, 132-147. Elsevier. 